\title{
The productivity and frequency of use of verbs of English origin recently lexicalized in French, Quebec and Czech contexts
}

\section{[La productivite et la frequence d'emploi des verbes d'origine anglaise recemment lexicalises dans les contextes francais, quebecois et tcheque]}

\author{
Radka Mudrochova
}

DOI: 10.18355/XL.2019.12.01XL.08

\begin{abstract}
This article focuses on productivity and frequency of verbs of English origin recently lexicalized in Czech and French. We will particularly observe, for the latter language, the uses of Quebec and France. Based on three representative lexemes entered in the 2018 edition of the Petit Robert dictionary, counterparts of which were found in Czech, we will highlight the differences or similarities of use of these chosen English borrowings based on lexicographical data and tools of corpus linguistics.

Key words: anglicism, loanword, lexicalization, productivity, frequency
\end{abstract}

\section{Résumé}

Le présent article porte sur la productivité et la fréquence des verbes d'origine anglaise récemment lexicalisés en tchèque et en français. Nous observerons particulièrement, pour cette dernière langue, les usages du Québec et de France. En prenant appui sur trois lexèmes, représentatifs, entrés dans l'édition $2018 \mathrm{du}$ dictionnaire le Petit Robert, dont les équivalents ont été trouvés en tchèque, nous ferons ressortir les différences ou les ressemblances d'emploi de ces emprunts à l'anglais choisis en nous appuyant sur des données lexicographiques et des outils de la linguistique de corpus.

Mots-clés : anglicisme, emprunt, lexicalisation, productivité, fréquence

\section{Introduction}

L'objectif principal de cette contribution ${ }^{1}$ est de présenter les résultats d'une analyse des verbes d'origine anglaise qui ont été récemment lexicalisés en français tout en prenant en compte les spécificités de la variété du français québécois et de comparer cet échantillon de verbes avec la situation linguistique tchèque.

Avant de présenter les résultats eux-mêmes, nous trouvons nécessaire d'introduire la présentation par un cadre théorique, notamment en définissant les termes clés apparus dans le titre de l'article, pour ensuite pouvoir décrire la méthodologie de recherche suivie de l'analyse détaillée des mots choisis. L'étude s'achève par une comparaison de résultats issus de l'analyse en incluant des perspectives de recherches ultérieures possibles.

\section{Mot lexicalisé/lexicalisation}

Un mot lexicalisé, le premier terme clé de cette contribution, est un mot connu / reconnu avec un sens déterminé, qui fait partie des dictionnaires. Jean et Nicole Tournier (2009 : 210) définissent la lexicalisation comme une intégration dans le lexique répertorié et renvoient au schéma, évoqué dans l'article «Dynamique

\footnotetext{
${ }^{1}$ Le présent article s'inscrit dans le Projet Européen du Développement Régional « Créativité et adaptabilité comme conditions du succès de l'Europe dans un monde interconnecté » (No. CZ.02.1.01/0.0/0.0/16_019/0000734) et a bénéficié du soutien du projet de l'Université Charles «Progres Q10 : Le langage dans les changements de temps, d'espace et de culture ».
} 
lexicale » (cf. figure 1), reposant sur quatre ensembles distincts : le lexique réel, le lexique potentiel, le non-lexique et le xénolexique.

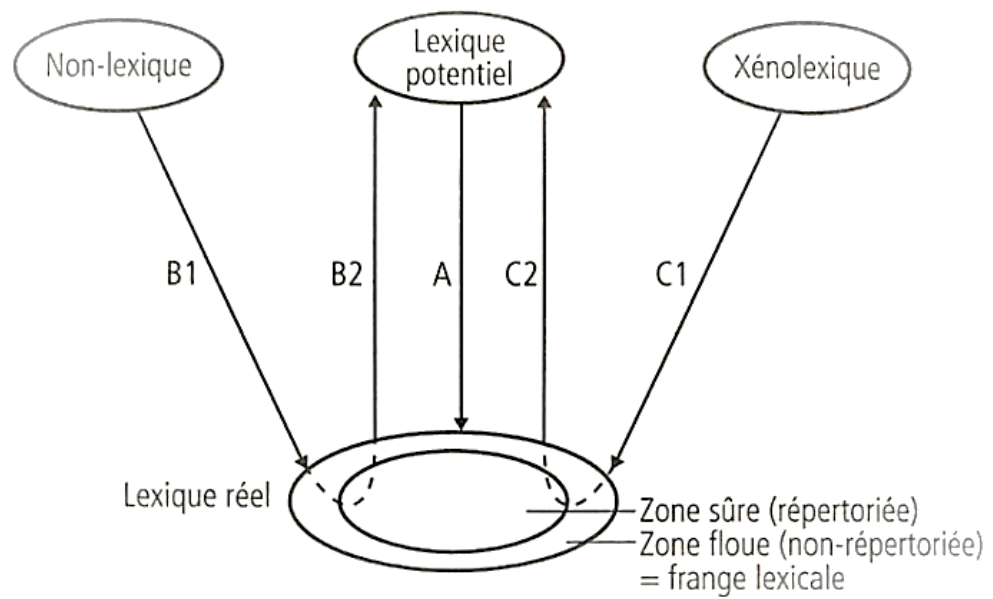

Figure 1 : Dynamique lexicale (Tournier 2009 : 118)

Le lexique réel est l'ensemble des lexies dans une langue qui distingue d'une part une «zone sûre » avec des lexies répertoriées et d'autre part une "zone floue », appelée aussi par Tournier (2009 : 117) frange lexicale, comprenant des lexies réalisées, mais non répertoriées. Le lexique non réalisé et non répertorié est intitulé le lexique potentiel. Le xénolexique est le lexique des autres langues et le non-lexique est représenté par des lexies impossibles dans une langue à un moment donné. Tous ces trois derniers ensembles cités peuvent servir de source pour le lexique réel. Les mots lexicalisés se trouvent donc dans la zone sûre du lexique réel, ils sont répertoriés et font donc partie des dictionnaires. Cette définition pourrait être comprise comme la définition contraire des néologismes dont la compréhension simplifiée repose sur le fait que le mot qui ne figure pas dans les dictionnaires peut être désigné comme un néologisme. Néanmoins, selon Sablayrolles (2017 : 14) cette simplification terminologique est insuffisante car elle pose plusieurs difficultés, notamment on peut se demander quel dictionnaire choisir comme arbitre ou si le mot se trouvant dans un dictionnaire perd automatiquement sa néologicité (?).Certes, il y a un lien direct entre la lexicalisation, comprise comme un processus d'intégration du lexique, et les néologismes car ces derniers représentent une source de la lexicalisation, cependant nous ne pouvons pas procéder à une simple opposition entre un mot lexicalisé et un néologisme, car un mot lexicalisé peut être perçu, pendant un certain temps et/ou sous certaines conditions, comme un néologisme et vice versa.

Pour les besoins de cette contribution, nous comprenons donc un mot lexicalisé comme un mot qui appartient au répertoire lexical des dictionnaires. Cependant il ne faut pas oublier que les corpus métalinguistiques reflètent l'usage des locuteurs finaux et que la lexicalisation est dans son ensemble un processus plus complexe.

\section{Anglicismes : bref rappel du contexte linguistique}

Un anglicisme, le second terme clé de cet article, dont le concept a fait déjà couler beaucoup d'encre au sein de la linguistique, est ici compris comme un mot ou locution 
d'origine anglaise utilisés, tels qu'ils sont ou avec modification de la forme (qu'elle soit graphique ou phonétique), dans une autre langue que de l'anglais ${ }^{2}$.

Évidemment, la présence et la perception des anglicismes sont différentes en français de France et en français québécois ainsi qu'en tchèque. Rappelons donc très brièvement les contextes linguistiques de ces trois territoires respectifs envers les emprunts à l'anglais.

La présence des emprunts anglais augmente dans le milieu tchèque au cours du $\mathrm{XX}^{\mathrm{e}}$ siècle pendant lequel le pays connaît trois vagues de leur adoption dans trois domaines distincts : le sport, la musique, la technique/l'électrotechnique. Pour le territoire tchèque il faut prendre en compte les barrières du régime communiste qui limitaient, voire même interdisaient, l'afflux de tout ce qui appartenait à l'Occident (cf. notamment Fidelius 2009 ; Mudrochová, Lazar 2017, 2018).

Quant à la France, ses échanges avec les territoires de parler anglais ont été plus nourris avec plusieurs vagues d'anglomanie comme le souligne Loubier (2011). Les linguistes Tournier (2009: 37) expliquent que «les emprunts à l'anglais britannique ont commencé à se multiplier sous le règne de la reine Victoria $[\ldots]$ au $\mathrm{XX}^{\mathrm{e}}$ siècle c'est de plus en plus à la variété américaine d'anglais que le français a emprunté [...]. ». Selon Rey (2008), les emprunts à l'anglais sont «parfaitement acceptés » jusqu'en 1940. Ainsi, c'est dans les années 50 qu'une vraie lutte contre l'anglomanie commence à se faire ressentir dans le discours des Français atteignant son sommet avec la parution du livre de René Étiemble « Parlez-vous franglais ? » daté de 1964.

Pour ce qui est de la situation au Québec, Loubier (2011: 18) indique qu'avant 1840, « les Canadiens français ne connaissent pas la honte linguistique », on se rend compte de l'anglicisation vers le milieu du XIX ${ }^{\mathrm{e}}$ siècle et entre 1940-1960 on parle déjà de la « chasse aux anglicismes ».

Pour terminer, il faut signaler que tandis qu'en France et au Québec il y a une politique linguistique de l'État qui régularise la présence des anglicismes dans les deux variétés du français en proposant des équivalents officiels et en commentant l'emploi inapproprié des alamodismes anglais, la République tchèque ne connaît aucune politique linguistique ni loi régularisant l'usage des emprunts.

\section{Méthodologie et étapes de la recherche}

Notre recherche ne comprend qu'un échantillon de mots, il s'agit des verbes d'origine anglaise, d'ailleurs dont le nombre ne cesse d'augmenter en français contemporain, entrés dans le dictionnaire le Petit Robert en 2018, donc récemment lexicalisés. Ces verbes sont au nombre de trois: googliser, liker et retweeter. Pour effectuer une analyse comparative, nous avons retrouvé les équivalents de ces trois lexèmes en tchèque et nous avons passé à une analyse plus détaillée qui respecte la méthodologie du projet EmpNéo (cf. Hildenbrand, Kacrpzak, Sablayrolles (éds) 2016 ; Kacprzak, Mudrochová, Sablayrolles (éds) 2019) enrichie par une recherche dans les corpus Aranea $^{3}$ (Benko 2018) et sur la plateforme Néoveille (Cartier 2016), étant, tous les deux, susceptibles de distinguer la variété québécoise, voire canadienne du français ${ }^{4}$.

\footnotetext{
${ }^{2}$ L'anglais, ici dans son sens le plus large, sans préciser le territoire de sa provenance pour ne pas être obligée de mettre par exemple à part les américanismes, etc.

${ }^{3}$ Les corpus Aranea comprennent 18 langues et sont composés des textes téléchargés automatiquement de différents sites Internet. La taille de ces corpus représente un avantage dans le fait que l'on a une base de données importante qui reflète le vrai usage de la langue de nos jours. D'un autre côté, les différents bruits que l'on rencontre lors des recherches peuvent être mis en cause.

${ }^{4}$ Dans cette contribution, nous employons les notions du français canadien et $\mathrm{du}$ français québécois comme des synonymes pour parler du français utilisé, pour nous
} 
Quant à la recherche dans les dictionnaires, nous avons utilisé les dictionnaires de langue générale traditionnels : le Petit Robert, Larousse; et électroniques : Usito (créé au Québec), Wiktionnaire/Wikipédia pour le français. Pour la langue tchèque nous avons travaillé avec Wikislovnik/Wikipedie, ABZ slovnik, Čeština 2.0, Slovník současné češtiny (Dictionnaire du tchèque contemporain) et la base Databáze heslář̀ qui comprend non seulement les dictionnaires généraux de la langue tchèque mais également la plateforme Neomat (un dictionnaire de néologismes créé par l'Institut pour la langue tchèque). Pour pouvoir nous prononcer sur la norme proposée par les instances de l'État, nous avons également consulté les dictionnaires terminologiques, celui de France: France Terme et celui du Québec: Grand dictionnaire terminologique. Pour la vérification de la fréquence et de la productivité, nous avons choisi d'une part les archives de presse de chaque territoire, 20minutes.fr pour le français de France, Lapresse.ca pour le français québécois et Idnes.cz pour la langue tchèque, et d'autre part le moteur de recherche Google limité à chaque fois au territoire concerné.

\section{Googliser/googlovat}

Le premier terme de notre recherche est représenté par le verbe googliser qui apparaît sous cette forme, donc en tant que l'entrée a priori du dictionnaire le Petit Robert, avec la définition : " rechercher des informations sur (qqch., qqn) sur Internet avec un moteur de recherche » dont l'étymologie renvoie à la marque déposée, Google, une marque américaine, et à la date de sa création, l'an 2003. Le Petit Robert cite également son concurrent formé par le suffixe -er, googler (daté de 2001). Le dictionnaire Larousse préfère, lui aussi, la variante googliser tandis que le dictionnaire québécois Usito celle de googler en notant qu'en France on emploie aussi googliser. Si le Petit Robert indique que le verbe googliser est un verbe transitif, le dictionnaire Usito contient aussi une définition suivie d'un exemple de l'emploi intransitif tout en étant plus précis sur le nom du moteur de recherche « faire une recherche sur Internet sur le moteur de recherche Google ». Pour ce qui est du Wiktionnaire, il comporte non seulement les deux variantes du verbe en question, faisant partie des verbes transitifs avec des datations de création mises entre parenthèses, googler (2007) et googliser (2011), mais ajoute aussi leurs dérivés, des verbes: auto-googliser, autogoogliser, dégoogliser, dé-googliser, regoogliser ; un nom masculin googlage (n.m.), un adjectif googlable et des synonymes ou plutôt des variantes orthographiques: google-iser, googoliser, gougueler, googueuliser, googleter.

En ce qui concerne les corpus Aranea (Araneum Francogallicum 8,70 G pour le français de France, AFM, et Araneum Francogallicum Canadiense 305 M pour le français canadien, AFC), ils affichent ces résultats respectifs (recherche effectuée le 29/09/2018) : en France 859 occ. de googler et 222 occ. de googliser, au Canada on reçoit un nombre moins important, étant donné que le corpus lui aussi est moins important que son homologue de France, googler 33, googliser 3. Quant aux dérivés, ils ne sont pas très représentés dans les corpus consultés, voici ses résultats, pour le AFM : auto-googliser (2), dé-googliser (2), dégoogliser (95), dégoogler (1), autogoogler (3), autogoogler (1), regoogler (1), googlage 32, googlable (10), et dans le AFC : auto-googler (1), googlable (2). En plus, le corpus AFM possède d'autres variantes orthographiques, de nouveau avec des fréquences peu importantes : googleiser (10), googoliser (9), googueler (1), googueuler (6), googueliser (5).

dans le discours écrit, au Canada sans spécifier ou distinguer explicitement chaque diversité territoriale.

XLinguae, Volume 12 Issue 1XL, January 2019, ISSN 1337-8384, eISSN 2453-711X 
Observons maintenant, grâce aux données illustrées par le tableau 1, les résultats de fréquence de googler et googliser dans les archives de presse, sur Google et sur la plateforme Néoveille.

\begin{tabular}{|l|l|l|l|l|l|l|}
\hline Mot & 20minutes.fr & Lapresse.ca & Google.fr & Google.ca & $\begin{array}{l}\text { Néoveille } \\
\text { FR }\end{array}$ & $\begin{array}{l}\text { Néoveille } \\
\text { CA }\end{array}$ \\
\hline googler & $44(2007)$ & $55(2007)$ & 38100 & 7640 & 49 & 13 \\
\hline googliser & $4(2013)$ & $1(2018)$ & 12000 & 221 & 47 & 0 \\
\hline
\end{tabular}

Tableau 1 : Fréquence d'emploi de googler et de googliser (FR vs CA)

Le tableau 1 fait ressortir plusieurs informations intéressantes, nous notons une plus grande fréquence d'emploi du verbe googler en France dont la datation reçue par les archives de 20minutes. fr remonte à une date plus lointaine dans l'histoire que pour googliser. Le même phénomène est à retenir pour le français canadien.

Pour googler ou googliser, la langue tchèque dispose d'un grand nombre d'équivalents proposés par le dictionnaire Neomat. D'une part, nous pouvons discerner les dérivés avec l'écriture d'origine (cf. tableau 2) et d'autre part, nous trouvons une variété de verbes avec des graphies tchéquisées (cf. tableau 3) tout en distinguant les verbes en -ovat en rouge et le groupe en -it en vert, les formes pronominales y compris, ainsi que les datations mentionnées par le dictionnaire, les plus anciennes sont mises en gras.

\begin{tabular}{|l|l|l|l|}
\hline googlovat & 2006 & googlit & 2014 \\
\hline googlovat se & 2013 & nagooglit & 2012 \\
\hline nagooglovat & 2010 & vygooglit & 2006 \\
\hline nagooglovat si & 2014 & vygooglit se & 2008 \\
\hline progooglovat & 2003 & zagooglit & 2006 \\
\hline progooglovat se & 2012 & zagooglit si & 2005 \\
\hline vygooglovat & 2002 & & \\
\hline zagooglovat & 2006 & & \\
\hline zagooglovat si & 2014 & & \\
\hline
\end{tabular}

Tableau 2 : Les verbes formés de Google avec la racine d'origine

\begin{tabular}{|l|l|l|l|l|}
\hline guglovat & $\mathbf{2 0 0 2}$ gůglovat & 2011 gúglovat & 2017 \\
\hline guglovat si & $\mathbf{2 0 0 2}$ gůglovat se & $\mathbf{2 0 0 7}$ vygúglovat & 2015 \\
\hline oguglovat & $\mathbf{2 0 0 2}$ vygůglovat & 2014 přegúglovat & $\mathbf{2 0 1 0}$ \\
\hline proguglovat & $\mathbf{2 0 0 2}$ zagůglovat si & $\mathbf{2 0 0 7}$ vygúglit & 2012 \\
\hline proguglovat se & $\mathbf{2 0 0 2}$ gůglit & 2012 gúglit & 2017 \\
\hline vyguglovat & $\mathbf{2 0 0 2}$ vygůglit & 2008 & \\
\hline vyguglit & 2013 & & \\
\hline
\end{tabular}

Tableau 3 : Les verbes formés de Google avec la graphie tchéquisée

En observant les deux tableaux, nous pouvons noter la date de la première apparition de la famille verbale de Google en tchèque, étant de 2002 non seulement pour les verbes avec la racine d'origine mais également pour un grand nombre de variantes avec la graphie tchéquisée guglovat.

En ce qui concerne d'autres dictionnaires tchèques, c'est encore le dictionnaire Čeština 2.0, créé par des internautes, qui contient plusieurs variantes du verbe googler en tchèque : googlit, googlovat, vygooglit, vygooglovat, gůglovat, guglit, vygůglit. 
Slovník současné češtiny en comporte deux : googlit, googlovat en les classant dans le groupe des verbes imperfectifs. Le Wikislovník et le dictionnaire $A B Z$ ne mentionnent aucune variante de googler/googliser en tchèque.

Le bilan de fréquence sélective se trouve dans le tableau 4.

\begin{tabular}{|l|r|r|r|r|}
\hline Variante orth. & Idnes.cz & Google.cz & Néoveille CZ & Araneum \\
\hline googlovat & $\mathbf{2 9}$ & $\mathbf{2 4} \mathbf{9 0 0}$ & $\mathrm{X}$ & $\mathbf{1 2 6 9}$ \\
\hline guglovat & 0 & 473 & 0 & 16 \\
\hline gůglovat & 1 & 1920 & 0 & 130 \\
\hline gúglovat & 0 & 119 & 0 & 3 \\
\hline googlit & 7 & $\mathbf{5 9 4 0 0 0}$ & 0 & 936 \\
\hline vygooglovat & $\mathbf{3 2}$ & $\mathbf{4 6 0 0 0}$ & $\mathbf{1}$ & $\mathbf{1 9 2 5}$ \\
\hline vyguglovat & 0 & 2040 & 0 & 43 \\
\hline vygůglovat & 0 & 3740 & 0 & 124 \\
\hline vygúglovat & 0 & 288 & 0 & 21 \\
\hline vygooglit & $\mathbf{1 3}$ & $\mathbf{5 9 4 0 0 0}$ & $\mathbf{2}$ & $\mathbf{2 7 3 0}$ \\
\hline
\end{tabular}

Tableau 4 : Bilan de fréquence (sélection)

Les verbes les plus productifs, d'après les résultats de la recherche effectuée (le 23 24/09/2018) dans les outils choisis, sont représentés par : googlovat, vygooglovat et vygooglit, autrement dit les verbes avec la graphie d'origine, un imperfectif googlovat et les deux autres perfectifs formés par le préfixe $v y$ - (ayant donc ici une fonction perfectivisante).

\section{Liker/likovat}

Le deuxième verbe choisi pour notre recherche est celui de liker, entré dans le Petit Robert en 2018, avec l'étymologie de 2010 et la définition: "anglic. indiquer que l'on apprécie (un contenu) sur un réseau social en cliquant sur le bouton prévu à cet effet ", et dans le dictionnaire Larousse en 2019. En tapant liker ou like dans le dictionnaire Usito, c'est la page " aimer qqch » avec l'entrée " j'aime » (substantivé) qui apparaît. Le Wiktionnaire propose aussi une entrée pour liker (depuis 2010) et révèle en même temps trois synonymes : aimer, plussoyer et poucer (celui-ci classifié de rare). La fréquence que nous avons observée non seulement pour le verbe liker, mais aussi pour ses synonymes suggérés (nous les avons mis en contexte du mot la page pour préciser leur emploi), est résumée par le tableau 5.

\begin{tabular}{|l|r|r|r|r|r|r|r|r}
\hline Équivalents & Araneum FR & Araneum CA & Néoveille FR & Néoveille CA & 20minutes.fr & Lapresse.ca & Google.fr & Google.ca \\
\hline liker & 3770 & 12 & 524 & 2 & 1010 & $14(2012)$ & 50300000 & 43700 \\
\hline liker la page & 490 & 2 & 5 & 0 & 737 & 0 & 247000 & 14100 \\
\hline aimer la page & 1545 & 34 & 3 & 0 & 2 & $1(2013)$ & 570000 & 22300 \\
\hline plussoyer & 363 & 0 & 1 & 0 & $2(2011)$ & 0 & 41000 & 263 \\
\hline poucer & 32 & 1 & 0 & 0 & 0 & 0 & 16500 & 1510 \\
\hline poucer la page & 0 & 0 & 0 & 0 & 0 & 0 & 2 & 0 \\
\hline plussoyer la page & 0 & 0 & 0 & 0 & 0 & 0 & 0 & 0 \\
\hline
\end{tabular}

Tableau 5 : Fréquence d'emploi du verbe liker et de ses synonymes

Les couleurs permettent de distinguer les deux contextes étudiés, le bleu pour le français et le rouge pour indiquer le contexte québécois. Tandis qu'en France nous notons une préférence d'emploi pour liker, au Canada, il y a une, légère, plus grande fréquence pour aimer (dans le contexte : "la page »). Le verbe plussoyer affiche un nombre encore considérable dans le corpus AFM ainsi que sur Google.fr et il est attesté dans les archives de presse 20minutes.fr, dont la première apparition est datée de 2011. En revanche, l'emploi de poucer n'est pas pour l'instant confirmé. 
Pour ce qui est de la langue tchèque, le Neomat possède sept verbes de la famille liker avec les datations mises par nous entre parenthèses : likovat (2011), lajkovat (2001), dislajkovat (2014), olajkovat (2011), ulajkovat (2017), ulajkovat se (2014), lajknout (2011). ${ }^{5}$ Nous trouvons des verbes imperfectifs sans (likovat) ou avec la graphie tchéquisée (lajkovat), par ailleurs, dont l'attestation est la plus ancienne dans le dictionnaire consulté, ainsi que des perfectifs formés par des préfixes comme olajkovat ou par une autre terminaison que -ovat, comme lajknout. Pour ce qui est des autres dictionnaires choisis pour l'analyse, le Wikislovník contient le nom lajk (like) en citant quatre dérivés, sans d'autres précisions, trois verbes lajkovat, olajkovat, lajknout et un autre nom lajkování. Quant au dictionnaire Čeština 2.0, il renvoie à quatre verbes, lajknout (2016), lajkovat (2015), polajkovat (2016), et ulajknout (2018) = faire un like involontaire - les deux derniers cités sont absents sur Neomat et les deux premiers cités sont notamment présents dans Slovník současné češtiny qui les classent d'argotiques. L'imperfectif lajkovat est également mentionné par ABZ slovník.

En vérifiant la fréquence d'emploi des formes de base (toutes datées de 2011 sur Neomat) dans le corpus Araneum, nous apprenons que la forme lajkovat a, avec 1743 occ., le plus de résultats, suivie de lajknout avec 112 occ. et likovat avec 66 occ. En observant les collocations à gauche et à droite (en position $-5 / 5 \log$ Dice) de la forme la plus fréquente du corpus (cf. tableau 6), nous remarquons que le verbe lajkovat est fréquemment cité dans le contexte d'autres verbes d'origine anglaise adaptés, morphologiquement (tweetovat, sharovat, postovat) et graphiquement (tvitovat, hejtovat, četovat) au système langagier tchèque. En plus, en troisième position, nous révélons le verbe plusovat étant un synonyme tchèque de lajkovat, correspondant à l'équivalent français plussoyer.

\begin{tabular}{|c|c|c|c|c|c|}
\hline & $\frac{\text { Cooccurrence }}{\text { count }}$ & $\frac{\text { Candidate }}{\text { count }}$ & $\underline{\text { T-score }}$ & $\underline{\text { MI }}$ & $\underline{\log D i c e}$ \\
\hline$\underline{P} \mid \underline{N}$ lajkovat & 19 & 1,743 & 4.358 & 14.981 & 7.480 \\
\hline$\underline{P} \mid \underline{N}$ Related & 17 & 2,246 & 4.122 & 14.455 & 7.125 \\
\hline$\underline{P} \mid \underline{N}$ plusovat & 5 & 23 & 2.236 & 19.299 & 6.535 \\
\hline$\underline{P} \mid \underline{N}$ tweetujte & 5 & 31 & 2.236 & 18.869 & 6.529 \\
\hline$\underline{P} \mid \underline{N}$ tweetovat & 5 & 287 & 2.236 & 15.658 & 6.334 \\
\hline$\underline{P} \mid \underline{N}$ četovat & 4 & 96 & 1.999 & 16.916 & 6.155 \\
\hline$\underline{P} \mid \underline{N}$ lajků & 8 & 1,935 & 2.828 & 13.583 & 6.155 \\
\hline$\underline{P} \mid \underline{N}$ postuje & 4 & 169 & 1.999 & 16.100 & 6.099 \\
\hline$\underline{P} \mid \underline{N}$ komentovat & 263 & 125,006 & 16.214 & 12.608 & 6.087 \\
\hline$\underline{\mathrm{P}} \mid \underline{\mathrm{N}}$ sdílet & 385 & 202,311 & 19.617 & 12.463 & 5.950 \\
\hline$\underline{P} \mid \underline{N}$ chatovat & 10 & 4,052 & 3.161 & 12.838 & 5.821 \\
\hline$\underline{P} \mid \underline{N}$ plusujte & 3 & 5 & 1.732 & 20.764 & 5.813 \\
\hline$\underline{P} \mid \underline{N}$ sharujte & 3 & 11 & 1.732 & 19.626 & 5.808 \\
\hline$\underline{P} \mid \underline{N}$ tvítovat & 3 & 13 & 1.732 & 19.385 & 5.806 \\
\hline$\underline{P} \mid \underline{N}$ hejtujte & 3 & 15 & 1.732 & 19.179 & 5.805 \\
\hline$\underline{P} \mid \underline{N}$ tweetujete & 3 & 30 & 1.732 & 18.179 & 5.792 \\
\hline
\end{tabular}

Tableau 6 : Collocations du verbe lajkovat dans le corpus AFM

Les vérifications de fréquence dans les autres sources choisies (Néoveille, Idnes.cz, Google.cz), n'ont pu que confirmer la productivité plus élevée de lajkovat (cf. tableau 7).

\footnotetext{
${ }^{5}$ Hana Prokšová décrit le verbe lajkovat dans son article daté de 2012 dans la revue « Naše řeč » (en ligne).
} 


\begin{tabular}{|l|r|r|r|}
\hline Variantes & Idnes.cz & Néoveille & Google.cz \\
\hline likovat & 0 & 1 & 32100 \\
\hline lajkovat & $\mathbf{2 3}$ & $\mathbf{7 2}$ & $\mathbf{9 0 8 0 0}$ \\
\hline lajknout & 3 & 0 & 41800 \\
\hline
\end{tabular}

Tableau 7 : Fréquence d'emploi des verbes likovat/lajkovat/lajknout

\section{Retweeter/retweetovat}

Le verbe retweeter, dernier exemple analysé, est entré dans le dictionnaire le Petit Robert en 2018 avec la définition «tweeter à nouveau (une information, un contenu) » et la note étymologique : « 2009 de re- et tweeter ». Il est également présent dans le Wiktionnaire (première mention en 2010) et dans le dictionnaire québécois Usito. Retweeter est dérivé du verbe tweeter, lui-même attesté par le Petit Robert en 2012 et par le Larousse en 2013.

Tandis que les dictionnaires créés en France ne contiennent aucun équivalent ni synonyme du verbe retweeter, le dictionnaire Usito en propose deux : "partager un gazouillis ", " retransmettre un gazouillis » en expliquant que retweeter (apparu en 2009 dans le Monde) ou sa variante graphique retwitter (apparu en 2009 dans la Libération), incluse aussi dans le dictionnaire en question, représentent des emplois critiqués au Québec : «l'emploi de retweeter, ou de sa variante retwitter, est critiqué comme synonyme non standard de partager un gazouillis, retransmettre un gazouillis. »

L'expression " partager un gazouillis » est aussi affichée sur le site de GDT, suggérée par L'Office québécois de la langue française en 2018.

Pour pouvoir nous prononcer sur l'emploi des lexèmes/expressions évoqués supra, nous avons effectué une recherche dans les corpus Aranea (30/09/2018), pour la France et pour le Québec séparément. En France c'est la forme retweeter $(873$ occ.) qui prédomine devant retwitter (173 occ.). Pour ce qui est de l'équivalent québécois, gazouillis, la simple requête de ce terme renvoie à d'autres contextes (cf. figure 2) comme bruit de voiture, chant des oiseaux, etc. 


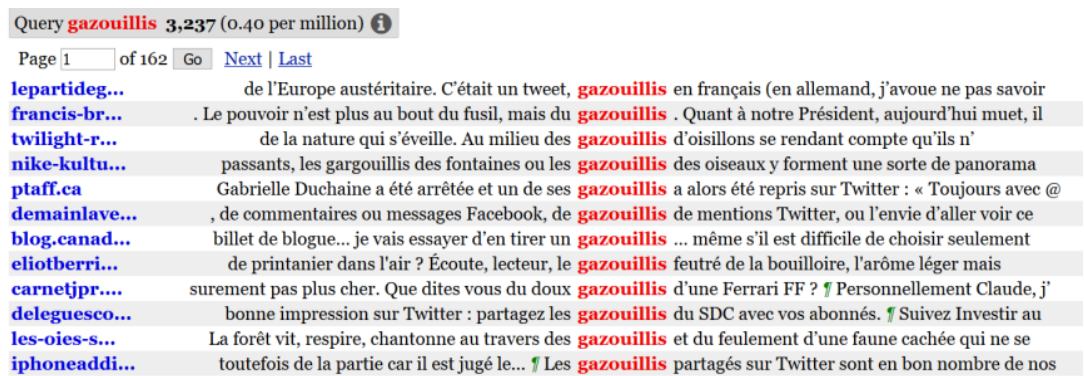

Figure 2 : requête gazouillis dans le corpus AFM

En précisant donc des expressions avec gazouillis pour le contexte de Twitter, nous recevrons « partager un gazouillis » avec 23 occ., " envoyer un gazouillis » (19 occ.) ou "publier un gazouillis » (16 occ.), donc un nombre non négligeable, mais moins élevé par rapport aux variantes accompagnées de tweet ${ }^{6}$.

Suite aux données du corpus Araneum, étudions maintenant l'usage au Québec où retweeter fournit 15 résultats et l'écriture retwitter qu'un seul. Le gazouillis affiche 439 occ. et si l'on précise le contexte par les verbes connus de la communication sur Twitter, nous obtiendrons ces chiffres : "partager» (22 occ.), « envoyer « 13 occ., « publier» $12 \mathrm{occ}$. Le taux de fréquence peut être exprimé en pourcentage par rapport à l'ensemble des données de gazouillis dans les deux contextes langagiers (cf. figure $3)$.

$\begin{array}{lcr} & \text { CA } & \text { FR } \\ \text { Partager } & 5 \% & 0,7 \% \\ \text { Envoyer } & 3 \% & 0,6 \% \\ \text { Publier } & 3 \% & 0,5 \%\end{array}$

Figure 3 : Comparaison de fréquence de gazouillis dans les corpus Aranea

La vérification de la fréquence sur la plateforme Néoveille confirme la préférence pour la graphie retweeter, 849 occ. en France contre 12 occ. pour retwitter. Tandis que la répartition de retweeter par territoire francophone est représentée par la figure 4 , la figure 5 illustre la répartition de gazouillis. Il faut noter que le contexte plus précis de Twitter n'a pas pu être pris en compte. Néanmoins en étudiant les résultats plus en détail, nous pouvons confirmer que le gazouillis apparaît dans les exemples de la presse canadienne plus souvent dans la signification tweet qu'en France.

\footnotetext{
${ }^{6}$ Par exemple « envoyer un tweet » apparaît en 326 cas.
} 

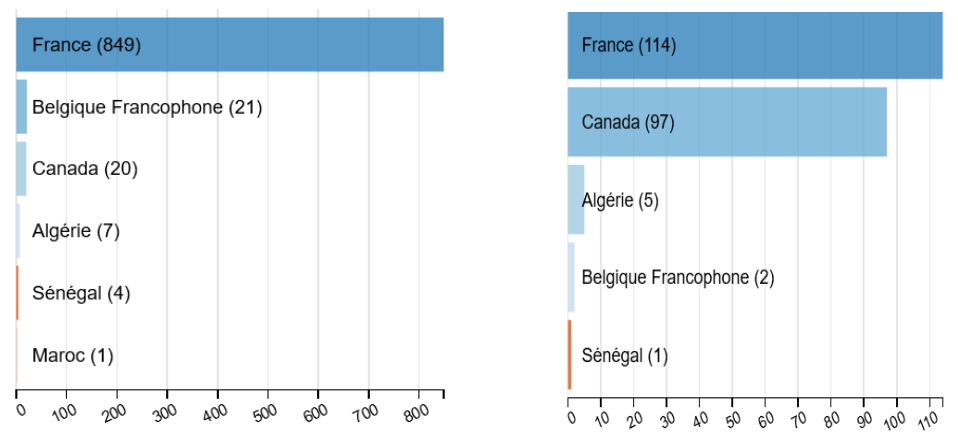

Figures 4 et 5 : Répartition de retweeter et de gazouillis sur Néoveille

Pour terminer cette analyse du verbe retweeter, voici le bilan de fréquence dans d'autres outils choisis (cf. tableau 8).

\begin{tabular}{|l|r|r|r|r|}
\hline Équivalents & 20minutes.fr & Lapresse.ca & Google.fr & Google.ca \\
\hline retweeter & $85(2009)$ & $34(2011)$ & 116000 & 10900 \\
\hline retwitter & $1(2016)$ & $4(2011)$ & 17100 & 1200 \\
\hline twitter "gazouillis" & $73(2009)$ & $736(2016)$ & 65000 & 21900 \\
\hline
\end{tabular}

Tableau 8 : Bilan de fréquence de la famille retweeter dans d'autres sources

En observant le tableau 8 nous remarquons que s'il y a en France, au moins dans les outils choisis, une plus grande fréquence d'emploi de retweeter, au Québec, nous observons des chiffres plus élevés pour gazouillis (en le mettant en même temps dans le contexte de twitter). D'un autre côté, nous n'osons pas dire qu'il s'agit d'une règle générale et qu'au Québec on suit les recommandations officielles indiquées car nous n'avons pas étudié en détail toutes les occurrences de gazouillis et notamment sa place pragmatique ou le son point de vue cognitif.

La langue tchèque, quant à elle, reconnaît dans son système deux équivalents de retweeter français, retweetovat (2013, imperfectif) et retweetnout (2014, perfectif) attestés respectivement, en 2013 et 2014, sur Neomat sans qu'il y ait une variante adaptée comme c'est le cas de tweeter (tweetovat), donc tvitovat daté de 2011 sur la plateforme évoquée. D'autres dictionnaires ne reflètent pas l'usage de ces formes, nous ne trouvons que tvítovat (daté de 2009) dans le dictionnaire des internautes, Čeština 2.0. Le bilan de fréquence de la famille retweeter en tchèque est illustré dans son ensemble par le tableau 9.

\begin{tabular}{|l|r|r|r|r|}
\hline \multicolumn{1}{|c|}{ Variantes } & Idnes.cz & Google.cz & Néoveille CZ & Araneum \\
\hline retweetovat & $\mathbf{4}$ & 1530 & 3 & $\mathbf{5 5}$ \\
\hline retweetnout & 0 & $\mathbf{4 7 9 0 0 0}$ & 0 & 31 \\
\hline retwittovat & 0 & 8 & 0 & 0 \\
\hline retwittnout & 0 & 3 & 0 & 0 \\
\hline retvitovat & 0 & 27 & 0 & 5 \\
\hline retvítnout & 0 & 0 & 0 & 3 \\
\hline
\end{tabular}

Tableau 9 : Bilan de fréquence de la famille retweeter en tchèque

D'après le tableau, c'est la forme imperfective retweetovat qui possède le plus de résultats dans les archives de presse Idnes, sur Néoveille et dans le corpus Araneum. En revanche, sur Google nous notons une fréquence plus importante pour la forme 
perfective retweetnout. La productivité des variantes tchéquisée restent plutôt marginale.

\section{Conclusion}

Pour conclure cette recherche des verbes d'origine anglaise récemment codifiés par le dictionnaire le Petit Robert, nous avons résumé les données lexicographiques sous forme de tableaux pour les deux langues respectives étudiées (tableau 10 et 11).

\begin{tabular}{|l|c|c|c|c|c|c|c|}
\hline Mot analysé & PR & Usito & Wiktionnaire & Larousse & FraTer & GDT & Datation \\
\hline googler & $\mathrm{O}$ & $\mathrm{O}$ & $\mathrm{O}$ & $\mathrm{O}$ & $\mathrm{X}$ & $\mathrm{X}$ & 2007 \\
\hline liker & $\mathrm{O}$ & $\mathrm{X}$ & $\mathrm{O}$ & $\mathrm{O}$ & $\mathrm{X}$ & $\mathrm{X}$ & 2010 \\
\hline retweeter & $\mathrm{O}$ & $\mathrm{O}$ & $\mathrm{O}$ & $\mathrm{X}$ & $\mathrm{X}$ & $\mathrm{OX}$ & 2009 \\
\hline
\end{tabular}

Tableau 10 : Résumé des données lexicographiques (googler, liker, retweeter)

\begin{tabular}{|l|c|c|c|c|c|c|}
\hline Mot analysé & Neomat & Čeština 2.0 & Abz slovník & Wikislovník & SSČ & Datation \\
\hline googlovat & $\mathrm{O}$ & $\mathrm{O}$ & $\mathrm{X}$ & $\mathrm{X}$ & $\mathrm{O}$ & 2002 \\
\hline lajkovat & $\mathrm{O}$ & $\mathrm{O}$ & $\mathrm{O}$ & $\mathrm{X}$ & $\mathrm{O}$ & 2001 \\
\hline retweetovat & $\mathrm{O}$ & $\mathrm{X}$ & $\mathrm{X}$ & $\mathrm{X}$ & $\mathrm{X}$ & 2013 \\
\hline
\end{tabular}

Tableau 11 : Résumé des données lexicographiques (googlovat, lajkovat retweetovat)

En observant les tableaux, il est à noter que le lexème googler est le plus présent dans les dictionnaires de la langue française et sa datation remonte jusqu'en 2007. En revanche, lajkovat, apparu en 2001, représente le mot le plus fréquent dans les dictionnaires de la langue tchèque.

Parlant des dictionnaires, il faut souligner que le tchèque dispose de la plateforme Neomat qui reflète plus rapidement les mots nouveaux, au contraire, Wikislovník reste très en retard par rapport au Wiktionnaire français et le dictionnaire Usito reste plus attentif envers les anglicismes (aimer, retweeter/retwitter). Ainsi, la politique linguistique, absente d'ailleurs en Tchéquie, fait naître, en France et au Québec, des recommandations officielles et des commentaires sur l'usage de tel ou tel mot dont l'usage réel n'est pas unanime.

Le verbe googliser, la forme de choix et de préférence des dictionnaires de langue générale (le Petit Robert, Larousse) ne correspond pas à la réalité de diffusion en français. En effet, notre analyse a confirmé la plus grande productivité de la forme googler, par ailleurs citée par Usito. Or, la langue tchèque dispose d'un grand nombre de variantes orthographiques dont l'usage n'est pas en réalité identique. Nous révélons trois variantes fréquentes : une forme de base googlovat (imperfectif) et deux dérivés (perfectifs) par préfixation (vy-) vygooglovat, vygoolit.

Le verbe liker, dont l'emploi est critiqué au Québec, connaît plusieurs synonymes évoqués par le Wiktionnaire, mais leur emploi en pratique n'a pas été confirmé par notre étude. Vu que le verbe liker est déjà attesté en tchèque en 2001, il y a une plus grande fréquence des adaptations orthographiques lajknout, lajkovat que des mots avec la racine d'origine (likovat).

Pour la lexie retweeter, étant la forme la plus répandue parmi d'autres graphies étudiées, le Québec dispose de deux expressions équivalentes recommandées, « partager un gazouillis », « retransmettre un gazouillis », dont l'emploi n'est pas de préférence en Belle province, mais n'est pas non plus négligeable.

Pour résumer, nous pouvons dire qu'en général, au Québec, on suit plus les recommandations d'emploi (liker vs aimer, retweeter vs partager un gazouillis) que sur le territoire français. Or, le tchèque, étant une langue flexive, a plus naturellement la tendance d'adapter les emprunts à son système langagier. 
Enfin, il est intéressant de suivre les mêmes traits de tendance pour les équivalents apparus dans les deux langues dont l'usage n'est pas de préférence, mais nous trouvons un certain nombre d'attestations : poucer $<$ opalcovat, plussoyer $<$ plusovat.

\section{Bibliographic references}

BLATNA, R. 200. K procesu prejimani slov (A propos des emprunts). In Cestina univerzalia a specifika 2. Brno : MU.

BOZDECHOVA, I. 1997. Vliv anglictiny na cestinu (L'influence de l'anglais sur le français). In F. Danes et al. (eds), Cesky jazyk na prelomu tisicileti. Praha : Academia, pp. 271-279.

CARTIER, E. 2016. Neoveille, systcme de reperage et de suivi des neologismes en sept langues, Neologica, n. 10, p. 101-131.

CERMAK, F. 2001. Jazyk a jazykoveda: prehled a slovniky (Langue, linguistique : presentation et dictionnaires). Praha : Karolinum

HILDENBRAND, Z. - KACPRZAK, A. - SABLAYROLLES J.-F. 2016. Emprunts neologiques et equivalents autochtones en français, en polonais et en tchcque, Limoges : Lambert Lucas.

JENKINS, J. 2004. ELF at the gate : The Position of English as a Lingua Franca. In The European English Messenger, vol. 13, n. 2, pp. 63-69.

KARLIK P. - NEKULA, M. - PLESKALOVA J. 2016. Novy encyklopedicky slovnik cestiny (Nouveau dictionnaire encyclopedique du tchcque en ligne), Available online : https://www.czechency.org/.

LOUBIER, Ch. 2011. De l'usage de l'emprunt linguistique. Montreal : Office quebecois de la langue française.

MARTINCOVA, O. et al. 1998. Nova slova $\mathrm{v}$ cestine. Slovnik neologizmu (Nouveaux mots en tchcque. Dictionnaire de neologismes). Praha : Academia.

MARTINCOVA, O. et al. 2002. Nova slova v cestine 2. Slovnik neologizmu (Nouveaux mots en tchcque 2. Dictionnaire de neologismes). Praha : Academia.

MUDROCHOVA R. - LAZAR, J. 2017. Vicejazycnost v kontextu jazykovych vypujcek - vliv francouzstiny a anglictiny na slovni zasobu $\mathrm{z}$ oblasti mody (Plurilinguisme dans le contexte des emprunts - l'influence du français et de l'anglais sur le vocabulaire tchcque issu de la mode), Sbornik konference Profilingua 14.19.9.2016, Plzen.

MUDROCHOVA R. - LAZAR, J. 2018. La circulation des emprunts neologiques dans le domaine de la mode en tchcque et en français dans une perspective diachronique, In ELAD-SILDA $\mathrm{n}^{\circ} 1$, Available online : http://revues.univlyon3.fr/elad-silda/index.php?id=324.

PROKSOVA, H. 2012. O novem ceskem slovese lajkovat (À propos d'un nouveau verbe tchcque liker). In Nase rec, vol. 95 n. 2, pp. 109-112, Available online : http://nase-rec.ujc.cas.cz/archiv.php?art=8243\#_ftn1.

SABLAYROLLES, J.-F. 2000. La neologie en français contemporain : examen du concept et analyse de productions neologiques recentes. Paris : H. Champion.

SABLAYROLLES, J.-F. 2017. Les neologismes. Creer des mots français d'aujourd'hui. Paris : Editions Garnier.

SOCHOVA Z. - POSTOLKOVA B. 1994. Co v slovnicich nenajdete. Novinky v soucasne slovni zasobe (Ce que vous ne trouverez pas dans les dictionnaires. Nouveautes dans le vocabulaire contemporain). Praha : Portal.

SVOBODOVA, D. 2007. Internacionalizace soucasne ceske slovni zasoby (Internationalisation du vocabulaire tchcque contemporain). Ostrava : OU.

SVOBODOVA, D. 2009. Aspekty hodnoceni cizojazycnych prejimek: mezi modnosti a standardem (Les aspects de l'evaluation des emprunts aux langues etrangcres : entre la modernite et le standard). Ostrava : OU.

XLinguae, Volume 12 Issue 1XL, January 2019, ISSN 1337-8384, eISSN 2453-711X 
TOURNIER, J. - TOURNIER, N. 2009. Dictionnaire de lexicologie française. Paris : Ellipses.

\section{Dictionnaries, corpus, on line platforms}

ABZSLOVNIK CIZICH SLOV, Available online : https://slovnik-cizich-slov.abz.cz/. BENKO V. 2018. Aranea: A Family of Comparable Gigaword Web Corpora, Web Corpora \& Corpus Linguistics Portal, Bratislava. Slovak Academy of Scientces L. Stur Institute of Linguistics, Available online http://aranea.juls.savba.sk/aranea_about/index.html.

BENKO, V. Araneum Bohemicum III Maximum, verze 17.04. Available online : http://unesco.uniba.sk/aranea/run.cgi/first?corpname=AranBohe_a\&reload=1.

BENKO, V. Araneum Francogallicum Canadiense, verze 18.03. Available online : http://unesco.uniba.sk/aranea/run.cgi/first?corpname=AranFrap_c\&reload=1.

BENKO, V. Araneum Francogallicum Maximum, verze 17.09. Available online : http://unesco.uniba.sk/aranea/run.cgi/first?corpname=AranFran_a\&reload=1

Cestina 2.0, Available online : https://cestina20.cz/.

DATABAZE HESLARU COMPORTANT : Prirucni slovnik jazyka ceskeho, Slovnik spisovneho jazyka ceskeho, Slovnik spisovne cestiny, Available online : http://lexiko.ujc.cas.cz/heslare/.

FRANCE TERME, Available online : http://www.culture.fr/franceterme.

LAROUSSE, Available online : https://larousse.fr/.

LE GRAND DICTIONNAIRE TERMINOLOGIQUE, Available online : http://www.granddictionnaire.com.

MOTS NOUVEAUX DES DICTIONNAIRES, Available online : http://www.orthogrenoble.net/mots-nouveaux-dictionnaires/.

NEOVEILLE, Available online : https://lipn.univparis13.fr/neoveille/html/login.php?action=login.

REY A. et al. 2019. Le Petit Robert. Paris: Petit Robert, Available online : https://pr.bvdep.com/robert.asp.

SLOVNIK SOUCASNE CESTINY, Available online : https://www.nechybujte.cz/slovnik-soucasne-cestiny.

Wikipedia, Available online : http://wikipedia.fr/.

Wikipedie, Available online : https://cs.wikipedia.org/.

Wikislovnik, Available online : https://cs.wiktionary.org/.

Wiktionnaire, Available online : https://fr.wiktionary.org/.

Idnes.cz, Available online : https://www.idnes.cz.

Lapresse.ca, Available online : https://www.lapresse.ca.

20minutes.fr, Available online : https://www.20minutes.fr.

Google Available online : https://www.google.com.

Words: 5021

Characters: 33328 (18,52 standard pages)

Radka Mudrochová, Ph.D.

Institute of Romance studies

Faculty of Arts

Charles University in Prague

Nám. Jana Palacha 2,

11638 Praha 1

Czech Republic

radka.mudrochova@ff.cuni.cz 